hep-th/9808055

CERN-TH-98-258

LPTHE-98-25

\title{
On Forms with Non-Abelian Charges and Their Dualities
}

\author{
Laurent Baulieu \\ CERN, Geneva, Switzerland \\ and \\ LPTHE⿴囗十, Paris, France.
}

\begin{abstract}
We describe forms with non-Abelian charges. We avoid the use of theories with flat curvatures by working in the context of topological field theory. We obtain TQFTs for a form and its dual. We leave open the question of getting gauges in which the form, or its dual, can be gauged away, in such way that the model has two dual formulations. We give the example of charged two-forms in six dimensions.
\end{abstract}

May 1998

1 UMR-CNRS associée aux Universités Pierre et Marie Curie (Paris VI) et Denis Diderot (Paris VII) 


\section{Introduction}

In this paper we show that non-Abelian interactions can be introduced rather straightforwardly for topological quantum field theories (TQFTs) of forms.

The natural generalization of the Abelian gauge symmetries for a $p$-form $B_{p}$ valued in some representation of a Lie algebra $G$ implies the introduction a Yang-Mills field $A$ together with $B_{p}$. One considers, as a first attempt, infinitesimal gauge transformations

$$
\begin{aligned}
& \delta A=d \epsilon+[A, \epsilon] \\
& \delta B_{p}=d \eta_{p-1}+\left[A, \eta_{p-1}\right]+\left[B_{p}, \epsilon\right] .
\end{aligned}
$$

Here, $\epsilon$ is the 0 -form parameter for the Yang-Mills symmetry, and $\eta_{p-1}$ is an infinitesimal $(p-1)$-form, valued in the same representation of $G$ as $B_{p}$.

It is only when the Yang-Mills curvature $F_{A}=d A+\frac{1}{2}[A, A]$ vanishes that the system of gauge transformations (1.1) closes. Moreover, the curvature $G_{p+1}=d B_{p}+\left[A, B_{p}\right]$ does not transform covariantly, except if $F=0$. This makes it difficult to construct an action invariant under the unrestricted set of transformations (1.1).

Some of the difficulties triggered by the non-closure of gauge transformations can be overcome by using the Batalin-Vilkoviski formalism [1]. Indeed, the latter is appropriate for gauge symmetries with infinitesimal gauge transformations, which only close modulo some equations of motion. It was already noticed in the past that, when one quantizes charged-forms, all relevant fields and antifields fit in a unifying formalism, which generalizes that of the genuine Yang-Mills case (see e.g. [2] and [3]). However, the invariant classical actions found in [3] were first order, and describe fields with vanishing curvatures. The situation was thus not quite satisfying, since one misses Lagrangians with squared curvature, that is, Lagrangians of the Yang-Mills-type. The fact that antifields and fields can be unified into objects which mix positive and negative ghost numbers, in a way that closely fits the idea of duality, was however quite encouraging.

Another difficulty that one also encounters with charged forms and their gauge transformations is that of their mathematical definitions. There is, however, a proposition that can be found in [4, which could be a hint to define non Abelian-forms and Yang-Mills fields in a unified geometrical framework. Following eq. (6.96) of 四, one can saturates the "colour" indices of the forms $B_{p}^{i}$ by matrices $S_{i}$ and extend the Lie algebra $G$ for the Yang-Mills field $A=T^{a} A^{a}$ as follows:

$$
\begin{aligned}
& {\left[T_{a}, T_{b}\right]=f_{a b}^{c} T_{c},} \\
& {\left[S_{i}, T_{a}\right]=r_{i a}^{k} S_{k},} \\
& {\left[S_{i}, S_{j}\right]=0 .}
\end{aligned}
$$


Then $B_{p}=B_{p}^{i} S_{i}$ and one defines the curvature $G_{p+1}=d B_{p}+\left[A, P_{b}\right]=G_{p+1}^{i} S_{i}$.

The idea that we develop here is that, if one allows general transformations for the $p$-form, while keeping track of the gauge symmetry (1.1) by considering the equivariant cohomology with respect to (1.1), we escapes almost by definition all problems raised by the non-closure of the transformations (1.1). The natural framework for this is TQFT. Thus, instead of (1.1), we consider the following system of infinitesimal transformations:

$$
\delta B=d \eta_{p-1}+\left[A, \eta_{p-1}\right]+\left[B_{p}, \epsilon\right]+\epsilon_{p}
$$

where the $p$-form parameter $\epsilon_{p}$ describes an infinitesimal arbitrary transformation for $B_{p}$. Indeed, arbitrary gauge transformations build a closed algebra. Thus, the presence of the parameter $\epsilon_{p}$ in the gauge transformations allows for a compensation for that part of gauge transformations that necessitates constraints to reach a closed algebra. By introducing an equivariant cohomology in the context of TQFT, it becomes possible to consistently isolate from the general transformations the reduced set of gauge transformations (1.1). One actually expects TQFTs whose actions are supersymmetric and contain squared curvatures for the forms.

The concept of degenerate gauge symmetries, which characterize TQFTs, must be applied to these gauge transformations: the number of parameters $\eta_{p-1}$ and $\epsilon_{p}$ for the symmetry defined by (1.3) exceeds the number of degrees of freedom of $B_{p}$. One can solve this difficulty, and distinguish between the gauge transformations with parameters $\eta_{p-1}$ or $\epsilon$ and the rest of the general transformations for $B_{p}$. This is an almost obvious generalization of the case of topological Yang-Mills symmetry [5].

It is actually simple to deduce from (1.3) a BRST operator which is nilpotent, independently of any constraint, and which consistently separates between the general gauge transformations and those tentatively defined by (1.1). The existence of such an operator eventually leads one to the existence of an invariant TQFT action, provided topological gauge functions exist for $B_{p}$.

For the sake of notational simplicity, we first consider the case of two-form gauge fields, $p=2$. Moreover, as for the construction of a TQFT action, we work in six dimensions as in the Abelian case analized in [6]. Afterwards, we generalize our formulae. For the general case, we introduce antifields, in a Batalin-Vilkoviski approach, which provides an interesting unification between all ingredients. We will consider the definition of a TQFT for a $p$-form and a $D-p-2$-form in $D$ dimensions. There is a natural question about knowing wether topological gauge functions exist such that the TQFT can be expressed solely in terms of the $p$-form or of the $(D-p-2)$-form, with duality transformations between the two formulations. We leave it unexplored. 


\section{The example of charged 2 -forms in six dimensions}

We call $B_{1}^{1}$ and $B_{0}^{2}$ the one-form ghost and zero-form ghost of ghost for the gauge symmetries of a charged two-form $B_{2}$. Then, $\Psi_{2}^{1}, \Phi_{1}^{2}$ and $\Phi_{0}^{3}$ are the topological ghost and ghosts of ghosts of $B_{2}$. All these fields are in the same representation of $G$ as $B_{2}$. (Following the conventional notation the upper index of a form will be its ghost-number and the lower one its ordinary form-degree.)

Let $D_{A} \cdot=d+[A, \cdot]$ be the covariant derivative with respect to $A$ and $F_{A}=D_{A} \wedge D_{A}=$ $d A+A \wedge A$ be the curvature of $A$. We define the curvature of $B_{2}$ as $G_{3}=D_{A} B_{2}$.

The topological BRST symmetry associated to the gauge symmetry (1.3) is defined from the expansion in ghost number of the following equations:

$$
\begin{gathered}
(s+d)(A+c)+\frac{1}{2}[A+c, A+c]=F_{A}, \\
(s+d)\left(B_{2}+B_{1}^{1}+B_{0}^{2}\right)+\left[A+c, B_{2}+B_{1}^{1}+B_{0}^{2}\right]=G_{3}+\Psi_{2}^{1}+\Phi_{1}^{2}+\Phi_{0}^{3} .
\end{gathered}
$$

( $c$ is the Faddeev-Popov ghost of $A$.) These equations define the action of $s$, with the desired property that $s^{2}=0$, if they are comparable with the Bianchi identity $D_{A} D_{A}=F_{A}$ and $D_{A} F_{A}=0$. Thus, we must define the action of $s+d$ on the fields of the right-hand side of (2.2) as:

$$
(s+d)\left(G_{3}+\Psi_{2}^{1}+\Phi_{1}^{2}+\Phi_{0}^{3}\right)+\left[A+c, G_{3}+\Psi_{2}^{1}+\Phi_{1}^{2}+\Phi_{0}^{3}\right]=\left[F_{A}, B_{2}+B_{1}^{1}+B_{0}^{2}\right] .
$$

This warranties that $(s+d)^{2}=0$, and thus $s^{2}=0$ on all fields.

One might be interested in a detailed expression of $s$ on the fields. It is obtained by expanding the BRST equations (2.2) and (2.3). One finds the following expression for the action of $s$ :

$$
\begin{gathered}
s B_{2}=\Psi_{2}^{1}-D_{A} B_{1}^{1}-\left[c, B_{2}\right] \\
s B_{1}^{1}=\Phi_{1}^{2}-D_{A} B_{0}^{2}-\left[c, B_{1}^{1}\right] \\
s B_{0}^{2}=\Phi_{0}^{3}-\left[c, B_{0}^{2}\right] \\
s \Psi_{2}^{1}=-D_{A} \Phi_{1}^{2}+\left[F_{A}, B_{0}^{2}\right]-\left[c, \Psi_{2}^{1}\right] \\
s \Phi_{1}^{2}=-D_{A} \Phi_{0}^{3}-\left[c, \Phi_{1}^{2}\right] \\
s \Phi_{0}^{3}=-\left[c, \Phi_{0}^{3}\right] .
\end{gathered}
$$

Of course, we have $s A=-D_{A} c$ and $s c=-\frac{1}{2}[c, c]$. No antifield dependence is necessary, since the general transformations described by $s$ determine a closed algebra. However, 
we will see in the next section how antifields can be consistently introduced in the BRST equations. We can already guess in a very elementary (although unnatural) way that antifields can be introduced by brute force in (2.4) and (2.5), by redefinitions of topological ghosts.

As for writing an action that is a classical topological invariant, and thus a starting point for a TQFT action ready for path integration, we need to specify the dimension of space. In six dimensions, we need a pair of two-forms, $B_{2}$ and ${ }^{c} B_{2}$, because $G_{3}$ has an odd degree (see [6] for the Abelian situation). The following action is a possibility, which generalizes the Abelian topological term $I_{c l}=\int_{6} d B_{2} \wedge d^{c} B_{2}$ in [6]:

$$
I_{c l}=\int_{6} \operatorname{Tr}\left(D B_{2} \wedge D^{c} B_{2}+F \wedge\left[{ }^{c} B_{2}, B_{2}\right]\right) .
$$

The doubling of the number of forms amounts to have mirror equations as in (2.2) and (2.3), with a duplication of all ghosts. (An index ${ }^{c}$ is introduced for all mirror fields.)

Using the techniques detailed in [6] (the only difference is that we must define the antighosts and Lagrange multipliers as elements of the same Lie algebra representation as $B_{2}$ ), one can impose in a BRST way the following choices of gauge functions for $B_{2}$ and ${ }^{c} B_{2}$ :

$$
D_{A[\mu} B_{\nu \rho]}+\epsilon_{\mu \nu \rho \alpha \beta \gamma} D_{A}^{\left[\alpha_{c}\right.} B^{\beta \gamma]}
$$

and for the ghosts and antighosts:

$$
\begin{array}{lcl}
D_{A}^{\nu} \Psi_{[\mu \nu]}^{1} ; & D_{A}^{\mu} \Phi_{\mu}^{2} ; & D_{A}^{\mu} \Phi_{\mu}^{-2} \\
D_{A}^{\nu c} \Psi_{[\mu \nu]}^{1} ; & D_{A}^{\mu c} \Phi_{\mu}^{2} ; & D_{A}^{\mu c} \Phi_{\mu}^{-2}
\end{array}
$$

The six-dimensional BRST invariant action which uses the gauge functions (2.7) and (2.8) is:

$$
\begin{aligned}
& \int_{6}\left|D_{A[\mu} B_{\nu \rho]}\right|^{2}+\chi^{\mu \nu \rho} D_{A[\mu} \Psi_{\nu \rho]}^{1}+\eta_{\mu}^{-1} D_{A}^{\nu} \Psi_{[\mu \nu]}^{1} \\
& \quad+X^{1} D_{A}^{\mu} \eta_{\mu}^{-1}+\Phi^{-3} D_{A \mu} D_{A}^{\mu} \Phi^{3} \\
& \quad+\Phi_{\mu}^{-2} D_{A \mu} D_{A}^{\mu} \Phi_{\nu]}^{2}+D_{A}^{\nu} \Phi_{\nu}^{-2} D_{A}^{\mu} \Phi_{\mu}^{2} \\
& \quad \text { - the same expression with all fields } \phi \text { replaced by }{ }^{\mathrm{c}} \phi .
\end{aligned}
$$

This action, which is an $s$-exact term, is the non-Abelian extension of that constructed in [6]. It possesses a genuine Yang-Mills invariance since all gauge functions in (2.7) and (2.8) are gauge-covariant under Yang-Mills transformations. The gauge-fixing of this symmetry 
is quite an obvious task. We can for instance go in a BRST invariant way to a FeynmanLandau-type gauge for $A$. We have also to gauge-fix the ordinary gauge invariance of the two-forms $B_{2}$ and ${ }^{c} B_{2}$. We must introduce the ordinary ghosts and ghosts of ghosts of $B_{2}$ and ${ }^{c} B_{2}$, and proceed by replacing all derivatives by covariant ones in the gauge functions of the Abelian case given in [6]. This can be done straightforwardly, and it gives additional ghost contributions to (2.9). In the next section, the ordinary ghosts of the two-forms will be introduced with a wider perspective.

Let us now stress that it is possible to couple the theory to a Yang-Mills TQFT instead to the genuine Yang-Mills theory. To do so, $F_{A}$ has to be replaced whereas it appears in (2.1), (2.2) and (2.3) by $F_{A}+\Psi_{1}^{1}+\Phi_{0}^{2}$. Here, $\Psi_{1}^{1}$ and $\Phi_{0}^{2}$ are the topological ghost and ghost of ghost of the Yang-Mills theory [7] [5], and thus (2.1) becomes:

$$
\begin{aligned}
(s+d)(A+c)+\frac{1}{2}[A+c, A+c] & =F_{A}+\Psi_{1}^{1}+\Phi_{0}^{2}, \\
(s+d)\left(F_{A}+\Psi_{1}^{1}+\Phi_{0}^{2}\right)+\left[A+c, F_{A}+\Psi_{1}^{1}+\Phi_{0}^{2}\right] & =0 .
\end{aligned}
$$

Then, $s A=\Psi_{1}^{1}-D_{A} c$ and $s c=\Phi_{0}^{2}-\frac{1}{2}[c, c]$, and eqs. (2.4) and (2.5) are modified accordingly.

Since the action (2.6) is a topological term, it is still invariant when the Yang-Mills field transforms as in (2.10). The gauge fixing for the two-form gauge field goes the same way as above, with the same gauge functions as in (2.7) and (2.8). However, new contributions come by varying the gauge field $A$, which is present in these gauge functions. This gives couplings between the topological ghost $\Psi_{1}^{1}$ of $A$ and the rest of the fields in $(2.9)$.

As for the dynamics of the Yang-Mills field, one can add to (2.6) an action $\int \operatorname{Tr} F_{\mu \nu} F^{\mu \nu}$ when the gauge symmetry of $A$ is defined by (2.1). On the other hand, when it is defined by (2.10), one can only add to (2.6) a topological Yang-Mills gauge action, as, for instance, the one defined in [8] for the six-dimensional case. There is another option (see next section), which consists in introducing a TQFT with an additional three-form, starting from the topological term $\int_{6} \operatorname{Tr} F_{A} \wedge D_{A} Z_{3}$. This provides an additional TQFT action of the Bogomolny type.

The important result is the existence of the action (2.9). It shows that we can define an invariant action depending on charged two-forms, with squared curvatures. Because it possesses a BRST invariance of the same type as the one of the Abelian case, its tree approximation is also related to Poincaré supersymmetry as in [6]. 
In order to look for observables of the TQFT, one remarks that the simplest possibility come from the cocycles that are defined by the ghost expansion of:

$$
\begin{array}{r}
\Delta_{6}=\operatorname{Tr}\left(\left(\mathrm{D}_{\mathrm{A}} \mathrm{B}_{2}+\Psi_{2}^{1}+\Phi_{1}^{2}+\Phi_{0}^{3}\right) \wedge\left(\mathrm{D}_{\mathrm{A}}{ }^{\mathrm{c}} \mathrm{B}_{2}+{ }^{\mathrm{c}} \Psi_{2}^{1}+{ }^{\mathrm{c}} \Phi_{1}^{2}+{ }^{\mathrm{c}} \Phi_{0}^{3}\right)\right. \\
\left.+F_{A} \wedge\left[{ }^{c} B_{2}+{ }^{c} B_{1}^{1}+{ }^{c} B_{0}^{2}, B_{2}+B_{1}^{1}+B_{0}^{2}\right]\right) .
\end{array}
$$

One has by construction that $(s+d) \Delta_{6}=0$, which implies $s \int_{\Gamma_{d}} \Delta_{d}^{6-d}=0$, where $0 \leq d \leq 6$, and the integration is done over a $d$-cycle $\Gamma_{d}$. The $\int_{\Gamma_{d}} \Delta_{d}^{6-d}$ are thus the candidates as observables of the TQFT. This generalizes the Yang-Mills situation [7][5]. However, we are not yet in a position to understand the meaning of these observables. It could be that they should only be considered for a gauge field $A$ with a vanishing curvature.

\section{Generalization: TQFTs for dual pairs}

We now wish to reach a more detailed understanding of the BRST structure introduced in (2.2) and (2.3), and (2.1) or (2.10). For this, the antifield-formalism of Batalin-Vilkoviski [1] turns out to be useful, not only for solving the questions related to the non-closure, but also for enlightening the idea of duality [3]. Introducing antifields is natural in various situations where the closure of gauge transformations holds only up to equations of motion (for example, see refs. [9]). It is also known that the four-dimensional Yang-Mills topological theory can be related to a non-Abelian $B-F$ system (see e.g. [10]). So, even in this simpler case, the use of antifields sheds a particular light on the theory.

In what follows, we introduce the relevant fields and antifields for quantizing (2.6) and construct a Batalin-Vilkoviski action. We will obtain at once the topological term (2.6) and the complete TQFT action with its antifield dependence. The latter defines the BRST symmetry equations by a master equation.

We can consider a more general case than that we have described above. Instead of $B_{2}$ and ${ }^{c} B_{2}$ in six dimensions, we introduce a $p$-form $U_{p}$ and a $(D-p)$-form $V_{D-p}$ in $D$ dimensions with the same charges under the Yang-Mills symmetry. We associate new fields to $U_{p}, V_{D-p-2}$ and the Yang-Mills field $A$ : they are respectively a $(D-p-1)$-form $X_{D-p-1}$, a $(p+1)$-form $Y_{p+1}$, and a $(D-2)$-form $B_{D-2}$. We also introduce a 2-form $W_{2}$ with its companion, a $(D-3)$-form $Z_{D-3}$. The forms $A, Z_{D-3}, B_{D-2}$, and $W$ are valued in $G$. The other fields are valued in a given representation of $G$ that can be chosen at will.

The TQFT that will be constructed will depend dynamically on the pair of fields $U_{p}$ and $V_{D-p-2}$. It is a very intriguing question to ask whether there exists different 
topological gauge functions that define TQFTs, which can be separately expressed solely in terms of $U_{p}$ or of $V_{D-p-2}$ (and supersymmetric partners), in such a way that the two formulations can be transformed into each other by duality transformations.

The justification for introducing all the other fields that accompany $A, U_{p}$ and $V_{D-p-2}$ can be found in [3]. Let us summarize the argument. One introduces "generalized forms" for which the degree is defined as the sum of the ghost number and ordinary form degree. Thus, a "generalized $p$-form" is made of a sum of forms, each of them having an ordinary form degree and an integer value for the ghost number, positive or negative, such that the sum of its ordinary degree and ghost number is equal to $p$. The ordinary form degree can only run from 0 to $D$. It follows that the expansion in ghost number of a "generalized $p$-form" always admits a finite number of terms, equal to $D+1$, independently of the value of $p$. Among these $D+1$ independent terms, there are forms with a positive ghost number and forms with a negative one. The former are ordinary ghosts, which can be interpreted as ghosts or ghosts of ghosts associated with the parameters of the (degenerate) gauge symmetry. By definition, the components with negative ghost numbers are antifields. All these fields are supposed to play a role in the TQFT. Since the ghost number of the antifield of a field with ghost number $g$ is equal to $-g-1$ [1], the antifield of a form $\phi_{q}^{g}$ in $D$ dimensions is a form $\psi_{D-q}^{-g-1}$. Thus, one can unify all the ghosts fields and the antifields for the quantization of a pair of classical forms $\phi_{q}$ and $\psi_{D-q-1}$ as the components of "generalized forms" $\tilde{\phi}_{q}$ and $\tilde{\psi}_{D-q-1}$, with degrees $q$ and $D-q-1$ respectively. The component $\phi_{q+g+1}^{-g-1}$ in the expansion of $\tilde{\phi}_{q}$ can be identified, for $g \geq 0$, as the antifield of the ghost $\psi_{D-q-g-1}^{g}$ in the expansion of $\tilde{\psi}_{D-q-1}$.

These remarks justify the introduction of all "companion" fields mentioned above for writing an action for the forms $A, U_{p}$ and $V_{D-p-2}$. Let us now write in more detail the ghost expansions of all these fields. For a better understanding, we write, in each pair of the following equations, fields and antifields on top of each other:

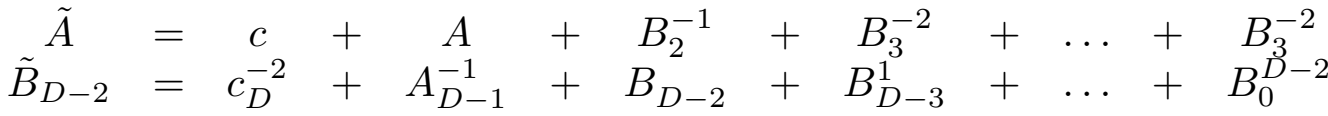

$$
\begin{aligned}
& \tilde{W}_{2}=\Phi_{0}^{2}+\Psi_{1}^{1}+W_{2}+Z_{3}^{-1}+Z_{4}^{-2}+\ldots+Z_{D}^{-D+2} \\
& \tilde{Z}_{D-3}=\Phi_{D}^{-3}+\Psi_{D-1}^{-2}+W_{D-2}^{-1}+Z_{D-3}+Z_{D-4}^{1}+\ldots+Z_{0}^{D-3}
\end{aligned}
$$




$$
\begin{aligned}
& \tilde{U}_{p} \quad=\quad U_{0}^{p}+\ldots+U_{p-1}^{1}+\quad U_{p}+X_{p-1}^{-1}+\ldots+X_{D}^{-D+p} \\
& \tilde{X}_{D-p-1}=U_{D}^{-p-1}+\ldots+U_{D-p+1}^{-2}+U_{D-p}^{-1}+X_{D-p-1}+\ldots+X_{0}^{D-p-1} \\
& \tilde{V}_{D-p-2}=V_{0}^{D-p-2}+\ldots+V_{D-p-3}^{1}+V_{D-p-2}+Y_{D-p-1}^{-1}+Y_{D-p}^{-2}+\ldots+Y_{D}^{-p-2} \\
& \tilde{Y}_{p+1}=V_{D}^{-D-p-1}+\ldots+V_{p+3}^{-2}+V_{p+2}^{-1}+Y_{p+1}+Y_{p}^{1}+\ldots+Y_{0}^{p+1}
\end{aligned}
$$

We recognize some the topological ghosts introduced in the particular case of the sixforms. For instance, the expansion of $\tilde{W}_{2}$ contains the topological ghosts $\Psi_{1}^{1}$ and $\Phi_{0}^{2}$ of $A$ and that of $\tilde{Y}_{p+1}$ contains the topological ghosts of $U_{p}$, which are identified as $Y_{p}^{1}, Y_{p-1}^{2}$, $\ldots, Y_{0}^{p+1}$. The antifield identification of the fields with negative ghost number will become quite obvious by looking at the BRST equations that we will shortly obtain, by imposing a Batalin-Vilkoviski type master equation.

We now introduce the TQFT Lagrangian density as the following $D$-form with ghost number zero:

$$
\begin{aligned}
\mathcal{L}_{D}=\operatorname{Tr}( & \tilde{X}_{D-p-1} \wedge \tilde{Y}_{p+1}+\tilde{X}_{D-p-1} \wedge D_{\tilde{A}} \tilde{U}_{p}+\tilde{Y}_{p+1} \wedge D_{\tilde{A}} \tilde{V}_{D-p-2} \\
& \left.+\tilde{B}_{D-2} \wedge \tilde{W}_{2}+\tilde{B}_{D-2} \wedge F_{\tilde{A}}+\tilde{W}_{2} \wedge\left(D_{\tilde{A}} \tilde{Z}_{D-3}+\left[\tilde{U}_{p}, \tilde{V}_{D-p-2}\right]\right)\right)\left.\right|_{D} ^{0}
\end{aligned}
$$

This Lagrangian is of first order and metric-independent. The intuition for writing (3.5) is that, if one considers its purely classical part $\mathcal{L}_{c l, D}$, by setting all ghosts and antifields equal to zero, (which means getting rid of all tildes in (3.5)), and if one eliminates $X_{D-p-1}$, $Y_{p+1}, B_{D-2}$ and $Z_{D-3}$ by their algebraic equations of motion $Y=D_{A} U, X=D_{A} V$ and $W=F_{A}$, we have:

$$
\begin{aligned}
\mathcal{L}_{c l, D} \sim \operatorname{Tr}( & D_{A} U_{p} \wedge D_{A} V_{D-p-2}+F_{A} \wedge\left[U_{p}, V_{D-p-2}\right] \\
& \left.+F_{A} \wedge D_{A} Z_{D-3}\right)
\end{aligned}
$$

As a result of the elimination of the fields $\tilde{X}$ and $\tilde{Y}$, the Lagrangian density (3.6) is locally $d$-exact, contrarily to (3.5). The integral over $D$-dimensional space of the density (3.6) can be considered as a topological term. Notice that the last term of (3.6) is $d$-exact by itself. In the particular case studied in the previous section, we had not introduced such a 
term in (2.6). However, we already observed that we had the freedom to consider arbitrary redefinitions of $A$ in (2.6), which is precisely the symmetry of $\int_{D} F_{A} \wedge D_{A} Z_{D-3}$.

We define the invariant Batalin-Vilkoviski-type action:

$$
\mathcal{I}_{D}=\int_{D} \mathcal{L}_{D}
$$

$\mathcal{I}_{D}$ satisfies a master equation, which is equivalent to its BRST symmetry:

$$
s \mathcal{I}_{D}=0, \quad s \phi=\frac{\delta \mathcal{I}_{D}}{\delta \psi}, \quad s \psi=\frac{\delta \mathcal{I}_{D}}{\delta \phi},
$$

where, generically, $\psi$ is the antifield of $\phi$.

Thus, the BRST equations for all fields and antifields defined in eqs. (3.1)-(3.4), can be obtained by varying (3.5). They are, in a compact form:

$$
\begin{gathered}
s \tilde{A}=-F_{\tilde{A}}+\tilde{W}_{2} \\
s \tilde{W}_{2}=-D_{\tilde{A}} \tilde{W}_{2} . \\
s \tilde{U}_{p}=-D_{\tilde{A}} \tilde{U}_{p}+\tilde{Y}_{p+1} \\
s \tilde{Y}_{p+1}=-D_{\tilde{A}} \tilde{Y}_{p+1} . \\
s \tilde{V}_{D-p-2}=-D_{\tilde{A}} \tilde{V}_{D-p-2}+\tilde{X}_{D-p-1} \\
s \tilde{X}_{D-p-1}=-D_{\tilde{A}} \tilde{X}_{D-p-1} . \\
s \tilde{Z}_{D-3}=-D_{\tilde{A}} \tilde{Z}_{D-3}+\left[\tilde{U}_{p}, \tilde{V}_{D-p-2}\right]+\tilde{B}_{D-2} \\
s \tilde{B}_{D-2}=-D_{\tilde{A}} \tilde{B}_{D-2}+\left[\tilde{X}_{D-p-1}, \tilde{U}_{p}\right]+\left[\tilde{Y}_{p+1}, \tilde{V}_{D-p-2}\right]+\left[\tilde{W}_{2}, \tilde{Z}_{D-3}\right] .
\end{gathered}
$$

(In order to reach a more detailed expression of $s$ on all fields and antifields, one has to do a further expansion in ghost number, as one does to obtain (2.4) and (2.5) from (2.2) and (2.3). For instance, (3.9) gives $s A=\Psi_{1}^{1}-D c, s c=\Phi_{0}^{2}-\frac{1}{2}[c, c]$, etc...)

Equations (3.9)-(3.12) are quite illuminating: if one sets all antifields equal to zero, one recovers field transformations similar to those in (2.2), (2.3) and (2.10); eqs. (3.9)(3.12) contain more information than eqs. (2.2) and (2.3) since they encode the way the antifields transform. This gives at once the BRST transformation, and a field and antifield dependent action, which we can gauge-fix by the Batalin-Vilkoviski method.

The transformations of $\tilde{A}, \tilde{U}, \tilde{V}$ and $\tilde{Z}_{D-3}$ show that these fields have a symmetry of the topological type. Indeed, the occurrence of $\tilde{W}, \tilde{Y}, \tilde{X}$ and $\tilde{B}_{D-2}$ in the right-handside of (3.9)-(3.12) implies a symmetry made of arbitrary shifts defined modulo gauge 
transformations. Moreover, the topological ghosts of $\tilde{A}, \tilde{U}, \tilde{V}$ and $\tilde{Z}_{D-3}$ are now identified as the ordinary ghosts and ghosts of ghosts of $W, Y, X$ and $B_{D-2}$. One can see the way the gauge symmetry is contained in the topological symmetry, by setting some of the topological ghosts equal to zero.

As already noted, the classical fields $W, Y, X$ and $B_{D-2}$ can be eliminated from the action because of their algebraic equations of motion. This operation gives the topological classical Lagrangian (3.6), plus antifield-dependent terms. By applying the BatalinVilkoviski procedure, these antifield-dependent terms eventually determine a fully gaugefixed action, which is analogous to (2.9). However, one needs to guess relevant gauge functions. The latter are expected to be of the self-dual type, as, for instance, those defined by (2.7) and (2.8), which are generically of the type $D_{A} U=*\left(D_{A} V\right)+\ldots$ Moreover, one can investigate the question of having more refined gauge functions, which would give a theory depending only on $U$ or $V$, with duality transformations between both formulations.

Let us conclude by the following remark. We can construct topological terms like (3.5). They are $D$-forms that can be written locally as $d$-exact terms. In the $D$-dimensional theory, and after the introduction of topological ghosts, they determines cocycles, analogous to those defined, in a particular case, in (2.11). These cocycles are candidates for observables in potentially interesting TQFTs. They also generate, by the descent equations for forms [11], consistent anomalies for the ordinary gauge symmetries of forms in $(D-2)$ dimensional field theories. 


\section{References}

[1] I.A. Batalin and V.A. Vilkowisky, Phys. Rev. D28 (1983) 2567;

M. Henneaux, Phys. Rep. 126 (1985) 1; M. Henneaux and C. Teitelboim, Quantization of Gauge Systems, Princeton University Press, Princeton (1992).

[2] L. Baulieu, E. Bergshoeff and E. Sezgin, Open BRST Algebra, Ghost Unification and String Field Theory, Nucl. Phys. B307 (1988) 348.

[3] L. Baulieu, Field Antifield Duality, p-Form Gauge Fields and Topological Quantum Field Theories, hep-th/9512026, Nucl. Phys. B478 (1996) 431.

[4] O. Alvarez, L. A. Ferreira and J. Sanchez Guillen, A New Approach to Integrable Theories in any Dimension, hep-th/9710147.

[5] L. Baulieu and I. M. Singer, Topological Yang-Mills Symmetry, Nucl. Phys. Proc. Suppl. 15B (1988) 12;

L. Baulieu, On the Symmetries of Topological Quantum Field Theories, hep-th 9504015, Int. J. Mod. Phys. A10 (1995) 4483;

R. Dijkgraaf and G. Moore, Balanced Topological Field Theories, hep-th/9608169, Commun. Math. Phys. 185 (1997) 411.

[6] L. Baulieu and P. West, Six-Dimensional TQFTs and Twisted Supersymmetry, hepth/9805200;

L. Baulieu and E. Rabinovici, Self-Duality and New TQFTs for Forms, hepth/9805122.

[7] E. Witten, Topological Quantum Field Theory, hep-th/9403195, Commun. Math. Phys. 117 (1988) 353.

[8] L. Baulieu, H. Kanno and I. Singer, Special Quantum Field Theories in Eight and Other Dimensions, hep-th/9704167, Talk given at APCTP Winter School on Dualities in String Theory (Sokcho, Korea), February 24-28, 1997;

L. Baulieu, H. Kanno and I. Singer, Cohomological Yang-Mills Theory in Eight Dimensions, hep-th/9705127, to appear in Commun. Math. Phys.

[9] L. Baulieu, M. Bellon, S. Ouvry and C. Wallet, Phys.Letters B252 (1990) 387; M. Bocchichio, Phys. Lett. B187 (1987) 322 and B 192 (1987) 31; R. Thorn, Nucl. Phys. B257 (1987) 61.

[10] G. Thompson, Annals Phys. 205 (1991) 130; J.M.F. Labastida and M. Pernici, Phys. Lett. 212B (1988) 56; D. Birmingham, M.Blau, M. Rakowski and G. Thompson, Phys. Rep. 209 (1991) 129.

[11] L. Baulieu, Algebraic Quantization of Gauge Theories, Lectures given at the Cargèse Summer School on "Perspectives in fields and particles", 198, p1, Proc. ed. by J.L. Basdevant and M. Levy (Plenum Press, New-York, 1983). 